\title{
2-hydroxycaproate predicts cardiovascular mortality in patients with atherosclerotic disease
}

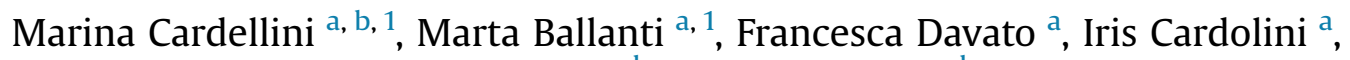 \\ Valeria Guglielmi ${ }^{\text {a, Stefano Rizza }}{ }^{\text {a, }}$, Chiara Pecchioli ${ }^{b}$, Viviana Casagrande ${ }^{\text {a }}$, \\ Maria Mavilio ${ }^{\mathrm{a}}$, Ottavia Porzio ${ }^{\mathrm{c}}$, Jacopo M. Legramante ${ }^{\mathrm{a}}$, Arnaldo Ippoliti ${ }^{\mathrm{c}}$, \\ Alessio Farcomeni ${ }^{\mathrm{d}}$, Paolo Sbraccia ${ }^{\mathrm{a}}$, Rossella Menghini a , Marc E. Dumas e, \\ Ben A. Kappel a, f, **, 2 , Massimo Federici a, b, *,2

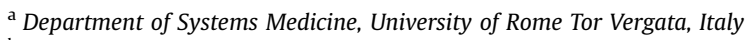 \\ ${ }^{\mathrm{b}}$ Center for Atherosclerosis, Policlinico Tor Vergata, Rome, Italy \\ ${ }^{c}$ Department of Experimental Medicine and Surgery, University of Rome Tor Vergata, Italy \\ ${ }^{\mathrm{d}}$ Department of Public Health and Infectious Diseases, University of Rome La Sapienza, Italy \\ e Imperial College, London, UK \\ ${ }^{\mathrm{f}}$ Department of Internal Medicine 1, University Hospital Aachen, RWTH Aachen University, Germany
}

\section{A R T I C L E I N F O}

\section{Article history:}

Received 24 February 2018

Received in revised form

24 May 2018

Accepted 8 June 2018

Available online 12 June 2018

\section{Keywords:}

Biomarker

Metabolomics

Cardiovascular mortality

Atherosclerosis

2-hydroxycaproate

\begin{abstract}
A B S T R A C T
Background and aims: We aimed to identify novel biomarkers for cardiovascular mortality through a non-targeted metabolomics approach in patients with established atherosclerotic disease from the Tor Vergata Atherosclerosis Registry (TVAR).

Methods: We compared the serum baseline metabolome of 19 patients with atherosclerosis suffering from cardiovascular death during follow-up with the baseline serum metabolome of 20 control patients matched for age, gender, body mass index (BMI) and atherosclerotic disease status, who survived during the observation period.

Results: Three metabolites were significantly different in the cardiovascular mortality (CVM) group compared to controls: 2-hydroxycaproate, gluconate and sorbitol. 2-hydroxycaproate (otherwise known as alpha hydroxy caproate) was also significantly correlated with time to death. The metabolites performed better when combined together rather than singularly on the identification of CVM status. Conclusions: Our analysis led to identify few metabolites potentially amenable of translation into the clinical practice as biomarkers for specific metabolic changes in the cardiovascular system in patients with established atherosclerotic disease.
\end{abstract}

๑) 2018 Elsevier B.V. All rights reserved.

\section{Introduction}

Cardiovascular events on the basis of atherosclerotic vascular disease (AVD) are the leading cause of mortality in the world [1]. Although many risk factors for AVD have been discovered, such as hyperlipidemia, hypertension, family background, smoking and

\footnotetext{
* Corresponding author. Via Montpellier 1, 00133, Rome, Italy.

** Corresponding author. Pauwelsstr. 30, 52074, Aachen, Germany. E-mail addresses: bkappel@ukaachen.de (B.A. Kappel), federicm@uniroma2.it (M. Federici).

1 Equally contributing first authors.

2 Equally contributing senior authors.
}

diabetes mellitus, $40 \%$ of patients with atherosclerosis have only one conventional risk factor and $20 \%$ of patients have none [2]. In addition, classical risk score calculators such as the Framingham risk score take only account of patients with unknown AVD status. However, it is particularly important to identify high risk patients with established AVD to develop individual treatment and preventative strategies.

A promising tool to identify high-risk patients might be the study of metabolite profiles in biological samples, especially in blood, so called "metabolomics". "Metabolome" refers to the set of all biochemical smaller than $2 \mathrm{kDa}$, including amino acids, sugars and lipids that can be found in biological samples [3]. Compared to the human proteome, which comprises more than $10^{6}$ molecules, it 
has recently been estimated that the human metabolome only contains approximately 3000 particles, making it easier to analyze than the proteome [4].

Metabolomics has been used to identify biomarkers for cardiovascular prediction in different cohorts [5-7]. For instance, fasting plasma trimethylamine $\mathrm{N}$-oxide (TMAO) levels, a gut microbiotadependent metabolite, was associated with coronary artery disease, stroke and heart failure in several studies [8]. Metabolites involved in pyrimidine metabolism, tricarboxylic acid (TCA) cycle, and the pentose phosphate pathway were altered after planned myocardial infarction (MI) [9]. Other cross-sectional and prospective studies led to identify phospholipids, fatty acids and amino acids as biomarkers for increased risk of cardiovascular and cerebrovascular events $[10,11]$.

We recently applied targeted-mass-spectrometry-based profiling of 49 metabolites, including amino acids and acylcarnitines, in a group of very old subjects [12]. We found that long-chain acylcarnitines, and alanine were independently associated with major adverse cardiac events (MACE) after adjustment for clinical cardiovascular covariates, suggesting that specific metabolites could help improve the identification of at risk subjects. Through a non-targeted mass-spectrometry-based profiling to identify pathways associated with cardiovascular disorders in experimental models, we found that alterations in metabolites connected to muscle breakdown, collagen/elastin, energy metabolism and branched-chain amino acids are potential disease biomarkers $[13,14]$.

The aim of the current study was to use the same non-targeted metabolomics approach to identify novel biomarkers for cardiovascular mortality in patients with established atherosclerotic disease from the Tor Vergata Atherosclerosis Registry (TVAR) [15].

\section{Materials and methods}

\subsection{Description of patients}

The TVAR observational registry study (ISRCTN registry, ID ISRCTN42405215) was previously described [15]. Subjects with established atherosclerotic disease were enrolled from 2007 at the Center for Atherosclerosis of the Policlinico Tor Vergata in Rome. All the patients undergone a vascular procedure for significant vascular stenosis or have had a major cardiovascular event. Subjects were excluded if they had liver disease, renal insufficiency, heart failure, coagulopathy or any other severe systemic or infectious disease. We yearly screened cardiovascular mortality, death for any other cause, survival and events by phone interview. In 2014, we recorded 66 non-fatal cardiovascular events (24.6\%) and 44 deaths (16,5\%), 20 of which were due to cardiovascular causes (7,5\%). 19 patients from the 20 male individuals with atherosclerotic disease suffering from cardiovascular death were included in the current study as "cardiovascular mortality" (CVM) group; 1 patient was excluded from the study because no serum was available for the metabolomics analysis. The control group matched the CVM group for age, body mass index (BMI), gender, lipid and inflammatory profile using unpaired Student $t$-test analysis, and was composed by 20 male subjects with chronic atherosclerotic disease, who survived the observation period. The mean observation time was $43.17 \pm 24.9$ months and the two groups were matched for duration of follow-up (CMV group $36.4 \pm 23.5$ months vs. control group $50.4 \pm 24.5$ months, unpaired Student $t$-test, $p>0,05$ ).

Clinical biochemistry laboratory measurements and methods were previously described $[15,16]$.

\subsection{Informed consent and ethics committee approval}

An informed written consent was obtained from all participants. The study was approved by the local ethics committee and the reported investigations were carried out in accordance with the principles of the Declaration of Helsinki as revised in 2000.

\subsection{Metabolomics analysis}

Non-targeted metabolomics were performed on the Metabolon (North Carolina, USA) platform that has previously been described $[17,18]$.

In short, samples were inventoried and immediately stored at $-80^{\circ} \mathrm{C}$. Several recovery standards were added prior to the first step in the extraction process for quality control purposes. To remove protein, dissociate small molecules bound to protein or trapped in the precipitated protein matrix, and to recover chemically diverse metabolites, proteins were precipitated with methanol. The resulting extract was analyzed by 4 different methods: two separate reverse phase (RP) Ultrahigh performance liquid chromatography/Mass Spectroscopy (UPLC-MS/MS) with positive ion mode electrospray ionization (ESI), RP/UPLC-MS/MS with negative ion mode ESI, and hydrophilic interaction chromatography/UPLC-MS/MS with negative ion mode ESI.

Raw data was extracted, peak-identified and quality control

Table 1

Patient baseline characteristics.

\begin{tabular}{|c|c|c|c|}
\hline & Control & CVM & $p$-value \\
\hline $\mathrm{N}$ & 20 & 19 & \\
\hline Gender (M/W) & $20 / 0$ & $19 / 0$ & \\
\hline Age (years) & $73.35 \pm 3.88$ & $75.42 \pm 5.31$ & 0.171 \\
\hline BMI $\left(\mathrm{kg} / \mathrm{m}^{2}\right)$ & $25.84 \pm 2.84$ & $25.14 \pm 4.17$ & 0.554 \\
\hline Systolic blood pressure $(\mathrm{mmHg})$ & $132.0 \pm 14.63$ & $140.59 \pm 20.06$ & 0.142 \\
\hline Diastolic blood pressure ( $\mathrm{mmHg}$ ) & $79.75 \pm 9.52$ & $85.59 \pm 11.44$ & 0.105 \\
\hline Total cholesterol (mg/dl) & $181.9 \pm 36.93$ & $185.33 \pm 41.97$ & 0.79 \\
\hline HDL cholesterol (mg/dl) & $47.60 \pm 12.8$ & $42.83 \pm 14.73$ & 0.293 \\
\hline LDL cholesterol (mg/dl) & $106.0 \pm 32.28$ & $115.06 \pm 41.42$ & 0.46 \\
\hline Triglycerides (mg/dl) & $118.65 \pm 46.86$ & $163.06 \pm 86.40$ & 0.053 \\
\hline eGFR & $83.20 \pm 20.73$ & $72.92 \pm 23.75$ & 0.187 \\
\hline Fasting plasma glucose $(\mathrm{mg} / \mathrm{dl})$ & $97.95 \pm 9.53$ & $108.37 \pm 18.58$ & 0.038 \\
\hline Fasting plasma insulin $(\mathrm{uU} / \mathrm{ml})$ & $12.11 \pm 5.97$ & $13.79 \pm 10.34$ & 0.543 \\
\hline $\mathrm{HbA1c}(\mathrm{mmol} / \mathrm{mol})$ & $36.85 \pm 7.81$ & $41.28 \pm 7.91$ & 0.092 \\
\hline HOMA IR & $2.20 \pm 1.54$ & $3.26 \pm 3.03$ & 0.173 \\
\hline hs-CRP (mg/dl) & $4.88 \pm 5.86$ & $13.68 \pm 24.43$ & 0.22 \\
\hline diabetes status $(0 / 1 / 2 / 3)$ & $(9 / 9 / 1 / 1)$ & $(5 / 7 / 4 / 3)$ & 0.244 \\
\hline Smoking (no/yes/ex) & $(10 / 5 / 5)$ & $(5 / 7 / 7)$ & 0.277 \\
\hline Statins (no/yes) & $(9 / 11)$ & $(11 / 8)$ & 0.314 \\
\hline Fibrates (no/yes) & $(19 / 1)$ & $(19 / 0)$ & 0.464 \\
\hline Omega-3 (no/yes) & $(19 / 1)$ & $(18 / 1)$ & 0.774 \\
\hline ASA (no/yes) & $(7 / 13)$ & $(12 / 7)$ & 0.23 \\
\hline ACE-i (no/yes) & $(11 / 9)$ & $(10 / 9)$ & 0.507 \\
\hline ARB (no/yes) & $(14 / 6)$ & $(13 / 6)$ & 0.55 \\
\hline Beta blockers (no/yes) & $(20 / 0)$ & $(15 / 4)$ & 0.047 \\
\hline Calcium ch blockers (no/yes) & $(16 / 4)$ & $(14 / 5)$ & 0.465 \\
\hline Alpha blockers (no/yes) & $(15 / 5)$ & $(17 / 2)$ & 0.249 \\
\hline Diuretics (no/yes) & $(13 / 7)$ & $(15 / 4)$ & 0.271 \\
\hline
\end{tabular}

Clinical baseline characteristics of subjects suffering from cardiovascular death in the observational period ( $=\mathrm{CV}$ mortality $(\mathrm{CVM})$ group) and control group including surviving individuals matched for age, sex, and body mass index. Analysis by unpaired Student $t$-test and $\mathrm{X}^{2}$ test revealed significant differences $(p<0.05)$ in fasting glucose and use of betablockers and a trend $(p<0.01)$ towards different levels of triglycerides and glycated hemoglobin (HbA1c).

BMI: body mass index. HDL: high density lipoprotein. LDL: low density lipoprotein. eGFR: estimated glomerular filtration rate. HOMA IR: Homeostatic Model Assessment for Insulin Resistance. hs-CRP: high-sensitivity C-reactive protein. ASA: acetylsalicylic acid. ACE-i: angiotensin-converting-enzyme inhibitors. ACE-i (no/yes). ARB: angiotensin receptor blockers). Diabetes status (0: Normal glucose tolerance/1 Impaired glucose tolerance/2: new diagnosis of type 2 diabetes/3: History of type 2 diabetes). 
processed using Metabolon's hardware and software. Compounds were identified by comparison to library entries of purified standards or recurrent unknown entities.

\subsection{Statistical analysis}

Quantitative variables were expressed as mean \pm SD and categorical variables were presented as numbers. Patients baseline characteristics were analyzed by unpaired Student $t$-test and $\mathrm{X}^{2}$ test with IBM software SPSS Version 23. $p$-values $<0.05$ were considered as statistically significant. Multivariate analysis was performed to test the effect of beta-blockers and survival status.

Metabolomics analysis was performed using Metabolon platform and analyzed by program $\mathrm{R}$ (http://cran.r-project.org/) and MetaboAnalyst 4.0 (www.metaboanalyst.ca) [19].

Group separation was evaluated using orthogonal partial Least squares discriminant analysis (O-PLS-DA). Permutation was used as performance measurement [20].

Differences in metabolites between the groups were first analyzed by Welch's Two-Sample $t$-test. Significant $(p<0.05)$ KEGG pathway identified metabolites were next subject to pathway enrichment analysis by hypergeometric test. For pathway topology analysis, relative-betweeness centrality was applied.

For biomarker discovery, a more stringent test was performed. Wilcoxon rank test between control and CVM group corrected for multiple testing by false discovery rate approach (FDR) was used; metabolites with $p<0.05$ and $q<0.05$ (=FDR 5\%) were considered as statistically significant. Significance analysis of microarrays (SAM), another approach to correct for multiple testing, was applied to confirm the previous results.

Additionally, receiver operating characteristic (ROC) curve was calculated for a single or multiple metabolites to evaluate biomarkers for cardiovascular mortality [19]. For multivariate model including continuous clinical variables and metabolites, ROC curves are generated by Monte-Carlo cross validation (MCCV) using balanced sub-sampling. In each MCCV, two thirds (2/3) of the samples are used to evaluate the feature importance. The top 2, 3, 5, $10 \ldots 100$ (max) important features are then used to build classification models which are validated on $1 / 3$ of the samples left out. The procedures were repeated multiple times to calculate the performance and confidence interval of each model (classification method: Linear Support Vector Machine (SVM). Ranking method: area under Receiver Operating Characteristic (AUROC)) [19].

Pearson correlation between metabolites and time to death in the CVM group was calculated by GraphPad Prism 7.0.

\section{Results}

\subsection{Baseline characteristics}

Characteristics of cases of cardiovascular mortality and controls from the TVAR are showed in Table 1. Among the classical risk factors, CVM did not differ from controls in age, BMI, cholesterol levels, smoking and diabetes status. We observed that fasting glucose but not HbA1c was slightly but significantly $(p=0.038)$ elevated in CVM. Triglycerides were slightly higher in CVM compared to controls, while among drugs, a minor difference was observed only in the greater use of beta-blockers in CVM (Table 1).

\subsection{Non-targeted metabolomics}

Next, we took advantage of the metabolomics approach to identify factors associated with mortality. Metabolomics analysis revealed 773 identified compounds.

After data processing and normalization, we first applied O-PLSDA to evaluate separation of groups by metabolites (Supplementary
A

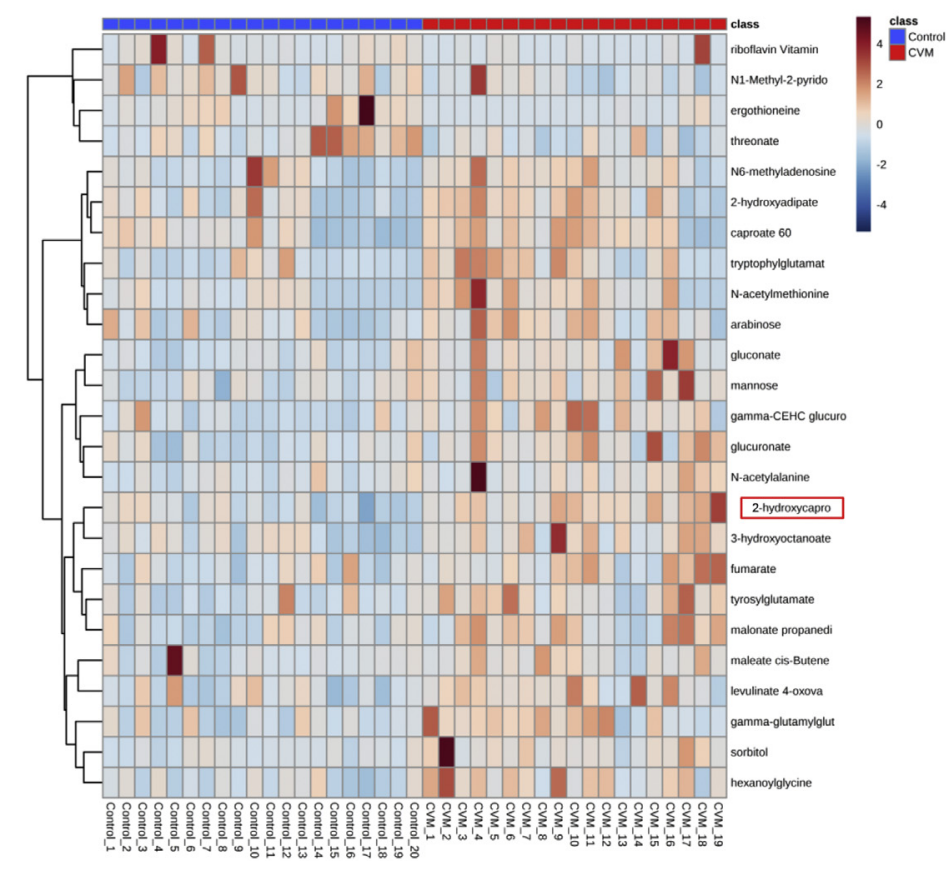

B

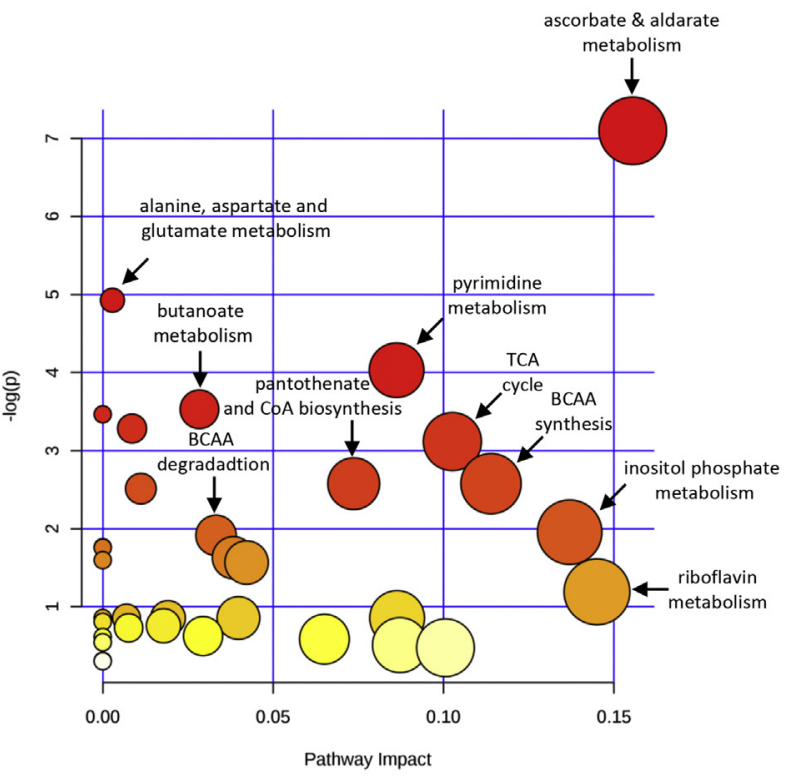

Fig. 1. Non-targeted metabolomics pathway analysis.

(A) Heatmap of non-targeted serum metabolomics showing the top 25 metabolites between cardiovascular mortality (CVM) and control group by Welch's Two-Sample t-test. (B) Pathway enrichment analysis by hypergeometric test of significant $(p<0.05)$ KEGG pathway identified metabolites reveal that several pathways differ between the CVM and control group, particularly, branched-chain amino acid (BCAA) metabolism, tricarboxylic acid (TCA) cycle and different co-factors such as ascorbate, pantothenate, coenzyme A (CoA) and riboflavin metabolism. 
Fig. 1). O-PLS-DA revealed a separation between the groups shown by a significant $\mathrm{Q}^{2}$ of 0.36 ( $p=0.001, \mathrm{n}=1000$ permutations).

Next, we used Welch's Two-Sample $t$-test to screen for metabolites and related pathways. We observed that a total of 74 metabolites were upregulated and 15 down-regulated in CVM compared to control $(p<0.05)$ (Supplementary Table 1 ). A heatmap reporting the 25 most relevant metabolites is shown in Fig. 1A.

Based on the significant metabolites between the groups, we performed additional pathway analyses (Fig. 1B; Supplementary Table 2), which revealed several differences between the two groups. Particularly, we found an impact on branched-chain amino acid (BCAA) synthesis and breakdown as well as TCA cycle.

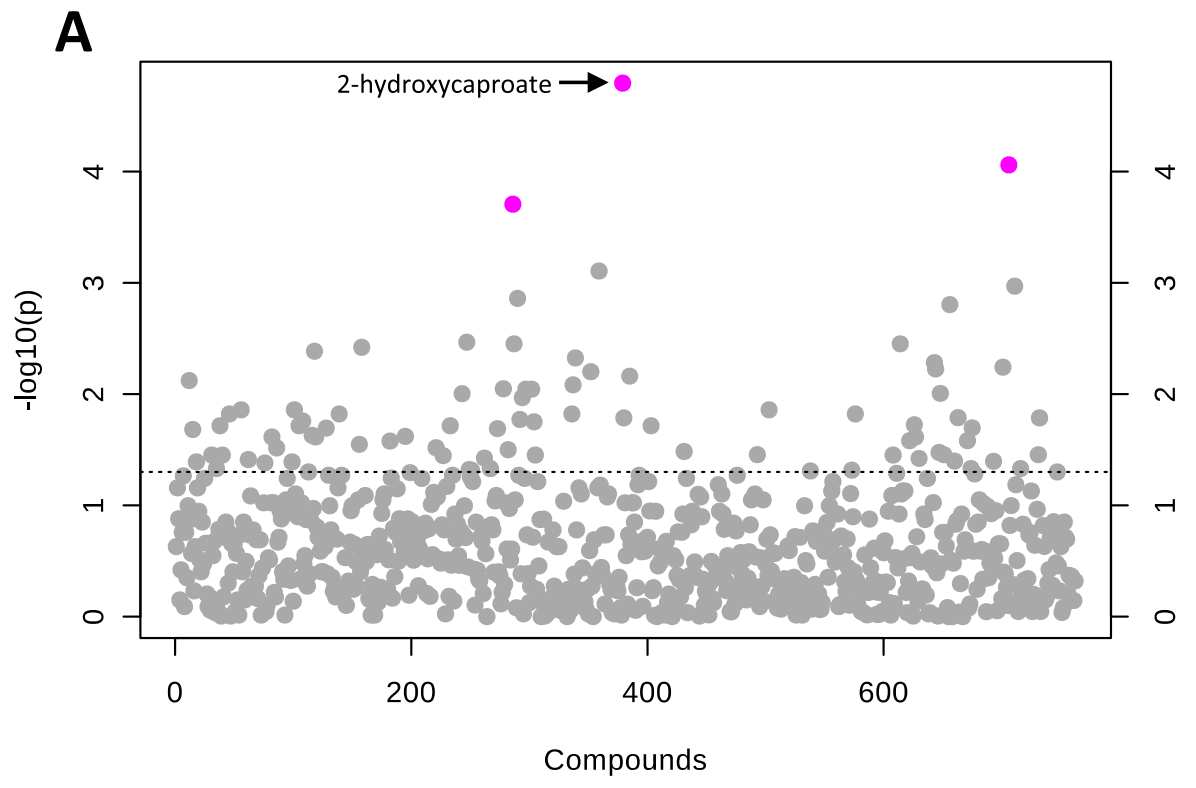

B

\begin{tabular}{llll} 
Metabolites & $\boldsymbol{p}$-value & $-\log 10(p)$ & q-value \\
\hline 2-hydroxycaproate & $1.60 \mathrm{E}-05$ & 4.796 & 0.012 \\
gluconate & $8.69 \mathrm{E}-05$ & 4.061 & 0.033 \\
sorbitol & $1.96 \mathrm{E}-04$ & 3.707 & 0.050 \\
\hline
\end{tabular}

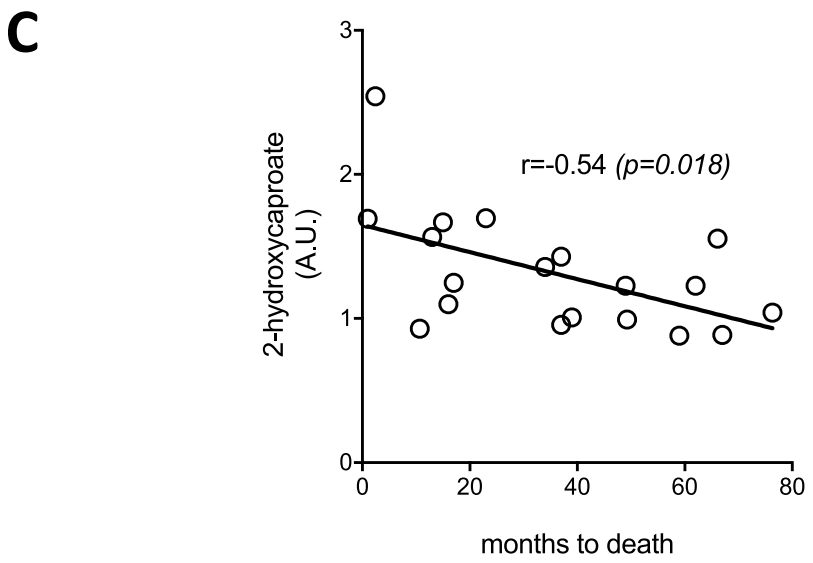

Fig. 2. 2-hydroxycaproate is the top metabolite to differentiate between groups and correlates with time to death.

(A and B) For biomarker discovery, a more stringent Wilcoxon rank test, corrected for multiple testing by false discovery rate (FDR=5\%) approach, was used, which revelaed 3 significant $(\mathrm{q}<0.05)$ metabolites: 2-hydroxycaproate, gluconate and sorbitol. 2-hydroxycaproate was identified as the most significant metabolite $(\mathrm{q}=0.012)$. (C) Pearson correlation analysis exposed significant correlation between levels of 2-hydroxycaproate and time to death in the cardiovascular mortality group ( $\mathrm{r}=-0.54$, $\mathrm{p}=0.018$ ), while no significant correlation between gluconate and sorbitol to time to death was found (data not shown). A.U.: arbitrary units. 
A
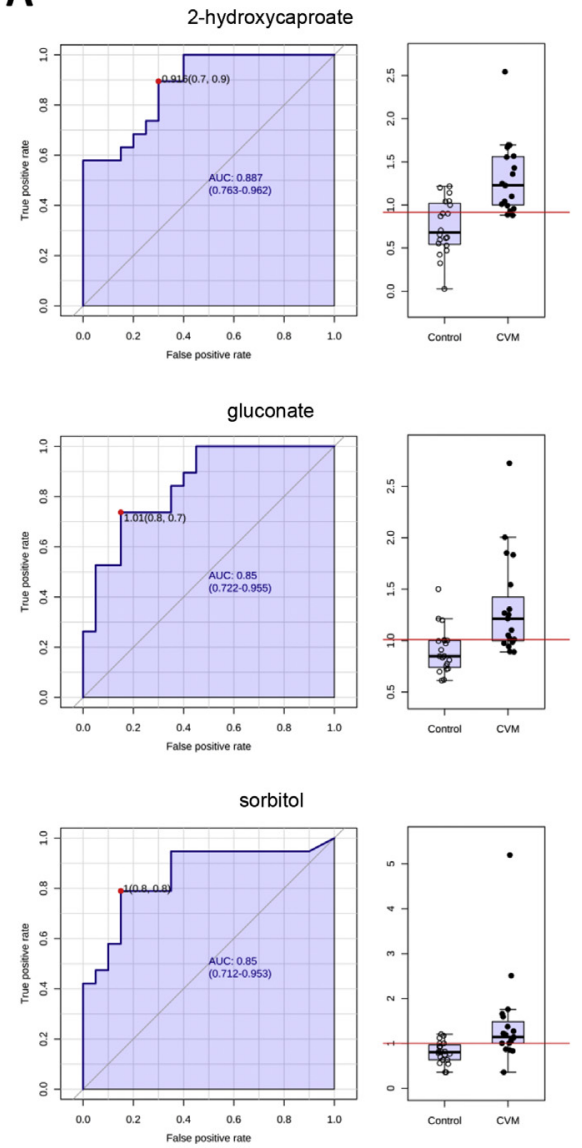

B



C

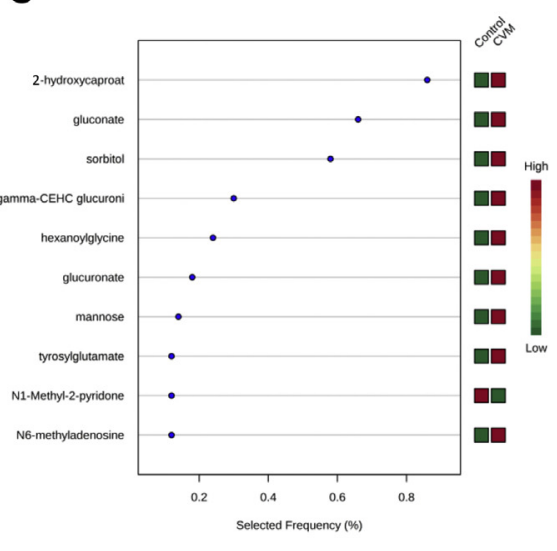

\section{D}



Fig. 3. Receiver operating characteristic analysis identifies 2-hydroxycaproate as top biomarker for cardiovascular mortality.

(A) Receiver operating characteristic (ROC) analysis was performed to evaluate metabolites as biomarkers for cardiovascular mortality. Single analysis of top 3 metabolites. (B) ROC curves generated with Monte-Carlo cross validation (MCCV). MCCV identified the 5 feature model as the best to discriminate between cardiovascular mortality group and control. (C) Top 10 selected frequency features showing 2-hydroxycaproate, gluconate and sorbitol as the most significant. (D) Combined ROC analysis of top 3 metabolite. AUC: Area under the curve; $95 \%$ confidence interval $(\mathrm{CI})$ in parentheses.

BCAA-derived metabolites included i.e. 4-methyl-2oxopentanoate (derived from leucine), 3-methyl-2-oxovalerate (derived from isoleucine), and 3-methyl-2-oxobutyrate (derived from valine) were observed in the CVM group. Reductions in circulating levels of additional metabolites derived from the BCAAs, including isovalerylcarnitine and tiglyl carnitine, were also observed in patients who experienced CVM (Supplementary Table 1).

Next, to focus on biomarkers rather than metabolic pathways, we applied a more stringent analysis using Wilcoxon rank test corrected for multiple testing by FDR 5\%. We found that only three metabolites were significantly different in CVM compared to controls: 2-hydroxycaproate (otherwise known as alpha hydroxy caproate), gluconate and sorbitol ( $p<0.05, \mathrm{q}<0.05$; Fig. 2A and B). 2-hydroxycaproate was also the most significant metabolite using different statistical approaches such as O-PLS-DA (Supplementary Fig. 1B) and SAM (Supplementary Fig. 2A and B). These three metabolites were significantly different between the two groups after an adjustment for use of beta blockers in multivariate analysis [2hydroxycaproate: beta $0.567(p<0.001)$; gluconate: beta 0.427 $(p=0.0019)$; sorbitol: beta $0.747(p=0.0045]$.

Pearson correlations revealed that levels of 2-hydroxycaproate correlated with time to death in the CVM group $(p=0.018$, $\mathrm{r}=-0.54$, Fig. 2C) while we did not observe any correlation with gluconate, sorbitol or other metabolites (data not shown).
Additionally, calculation of ROC curves was performed to evaluate the previous identified metabolites as biomarker for cardiovascular mortality. For 2-hydroxycaproate a high AUC value of 0.887 (95\% confidence interval (CI): $0.763-0.962$ ) was calculated, whereas for both gluconate and sorbitol the AUC was 0.85 (Fig. 3A).

Next, we generated a model including metabolites and continuous clinical variables. The MonteCarlo cross validation (MCCV) identified the 5 feature model as the best to discriminate between CVM and control (Fig. 3B). The selected frequency table of features identified 2-hydroxycaproate, sorbitol and gluconate as most picked features (Fig. 3C).

Analysis of AUC for the set of all three metabolites yielded an AUC of 0.907 (95\% CI: 0.737-1.0) (Fig. 3D).

\section{Discussion}

In this study, we evaluated the metabolome in subjects with established atherosclerosis and correlated it with clinical data and cardiovascular death to identify potential pathways and biomarkers to personalize risk reduction in subjects in secondary prevention.

Our analysis revealed that despite a robust class distinction (i.e. death against survival) and very mild clinical differences, metabolomics did not identify specific metabolic pathways pointing to specific biological processes that sustain the major clinical event.

However, metabolomics led to identify few metabolites 
potentially amenable to translation to clinical practice as biomarkers for specific metabolic changes in the cardiovascular system.

In our study, BCAA metabolism showed significant changes between the two groups. The branched chain amino acids (valine, isoleucine, and leucine) constitute a large portion of the amino acids found in muscle proteins, but are also readily released and metabolized by various tissues, including skeletal and cardiac muscle, to meet energy demands [21]. Interestingly, recent studies in the literature have uncovered a potential role for BCAA catabolism in cardiac pathophysiology and have established metabolic defects related to BCAA degradation as a contributing factor to cardiovascular disease [22]. In a process that occurs predominantly in mitochondria, BCAAs are first converted to their $\alpha$-keto acid derivatives by branched-chain amino transferase (BCAT). Branched-chain $\alpha$-keto acids are subsequently oxidized by the branched-chain $\alpha$-keto acid dehydrogenase enzyme complex (BCKD) to acetyl-CoA and succinyl-CoA, which can enter anabolic pathways (i.e., gluconeogenesis, fatty acid synthesis) and/or pathways of energy generation (i.e., TCA cycle). In a recent study using the same non-targeted metabolomics approach, we observed that treatment with empagliflozin enhances BCAA catabolism, thus potentially explaining its cardioprotective effect [23].

In the current study, we found 2-hydroxycaproate, a branched chain alpha-keto acid, significantly increased at baseline in subjects with cardiovascular mortality. Although directionality of change is difficult to decipher from static measurements, these changes may be suggestive of a defect in BCAA catabolism in the CVM group that is possibly related to mitochondrial dysfunction. In addition to the potential for altered energetics, changes in BCAA metabolism may modulate cardiovascular function through changes in m-TOR signaling. Alterations in BCAA catabolism observed in the current study provide additional evidence implicating this pathway in cardiovascular mortality in humans.

2-hydroxycaproate was previously observed in human blood and found increased in cerebrospinal fluid of patients affected by Nocardia meningitis [24,25]. 2-hydroxycaproate is among the inhibitors of aminoacylase 1, an hydrolase involved in several recycling and degradation pathways including conversion of $\mathrm{N}$-acyl- $\mathrm{L}-$ amino acids into L-amino acids in the kidney and in the regulation of the urea cycle [26]. Interestingly, in a recent study from the Framingham cohort an aptamer-based proteomic profile revealed aminoacylase 1 as a new biomarker for cardiovascular disease [27]. How the interaction between 2-hydroxycaproate and aminoacylase 1 affects cardiovascular function remains to be established. Our data, however, revealed a positive correlation between gluconate and hsCRP and fibrinogen (data not shown), suggesting a link to low grade inflammation, an important contributor to both metabolic and cardiovascular incident risk $[28,29]$ that needs further exploration.

Our study has limitations. The number of patients enrolled was low and the power of the study has not been calculated, therefore, our hypothesis-generating results must be validated in a larger cohort with similar characteristics. The use of beta blockers was lower in the CVM group, however, the metabolite was still significant after adjustment in multivariate analysis.

Future studies must also confirm if those biomarkers are suitable for different populations, including different ethnicities. If the predictive efficacy of these metabolites will be validated, it is mandatory to test their efficiency to improve risk scores using reclassification methods. This procedure was recently performed with low-grade inflammatory markers or metabolites using the Framingham Recurrent Score or the FINRISK Score [12,30,31].

\section{Conflicts of interest}

The authors declared they do not have anything to disclose regarding conflict of interest with respect to this manuscript.

\section{Financial support}

This research was in part funded by MIURPRIN 2015MPESJS_004 and Fondazione Roma Non Communicable Diseases 2014 Call to M.F. B.A.K. was supported by a grant from the Deutsche Herzstiftung.

\section{Author contributions}

M.F., B.A.K. conceived and supervised the study. M.C. interpreted results and generated tables. M.C., B.A.K., V.C., performed statistical analysis. M.F., M.C., M.B., B.A.K. wrote the manuscript. M.B., F.D., I.C., S.R., V.G., M.M., C.P., A.I. performed experiments. R.M., J.M.L., P.S. and M.E.D. contributed to data interpretation. All authors discussed the data and commented on the manuscript before submission.

\section{Appendix A. Supplementary data}

Supplementary data related to this article can be found at https://doi.org/10.1016/j.atherosclerosis.2018.06.014.

\section{References}

[1] M.H. Forouzanfar, L. Alexander, H.R. Anderson, V.F. Bachman, S. Biryukov, et al., Global, regional, and national comparative risk assessment of 79 behavioural, environmental and occupational, and metabolic risks or clusters of risks in 188 countries, 1990-2013: a systematic analysis for the global burden of disease study 2013, Lancet 386 (10010) (2015 Dec 5) 2287-2323, https:// doi.org/10.1016/S0140-6736(15)00128-2. Epub 2015 Sep. 11.

[2] A. Hozawa, A.R. Folsom, A.R. Sharrett, L.E. Chambless, Absolute and attributable risks of cardiovascular disease incidence in relation to optimal and borderline risk factors: comparison of African American with white subjects atherosclerosis risk in communities study, Arch. Intern. Med. 167 (2007) 573-579, https://doi.org/10.1001/archinte.167.6.573.

[3] A. Giovane, A. Balestrieri, C. Napoli, New insights into cardiovascular and lipid metabolomics, J. Cell. Biochem. 105 (3) (2008 Oct 15) 648-654, https:/| doi.org/10.1002/jcb.21875.

[4] R.E. Gerszten, T.J. Wang, The search for new cardiovascular biomarkers, Nature 451 (7181) (2008 Feb 21) 949-952, https://doi.org/10.1038/nature06802.

[5] L. Zhang, T.T. Wei, Y. Li, J. Li, Y. Fan, et al., Functional metabolomics characterizes a key role for N-Acetyl-Neuraminic acid in coronary artery diseases, $\begin{array}{llll}\text { Circulation (2017 Dec 6), https://doi.org/10.1161/ } & \end{array}$ CIRCULATIONAHA.117.031139.

[6] A. Ganda, L. Yvan-Charvet, Y. Zhang, E.J. Lai, R. Regunathan-Shenk, et al., Plasma metabolite profiles, cellular cholesterol efflux, and non-traditional cardiovascular risk in patients with CKD, J. Mol. Cell. Cardiol. 112 (2017 Nov) 114-122, https://doi.org/10.1016/j.yjmcc.2017.05.001. Epub 2017 May 4.

[7] J.R. Ussher, S. Elmariah, R.E. Gerszten, J.R. Dyck, The emerging role of metabolomics in the diagnosis and prognosis of cardiovascular disease, J. Am. Coll. Cardiol. 68 (25) (2016 Dec 27) 2850-2870, https://doi.org/10.1016/ j.jacc.2016.09.972.

[8] Y. Heianza, W. Ma, J.E. Manson, K.M. Rexrode, L. Qi, Gut microbiota metabolites and risk of major adverse cardiovascular disease events and death: a systematic review and meta-analysis of prospective studies, J Am Heart Assoc 6 (7) (2017 Jun 29), https://doi.org/10.1161/JAHA.116.004947 pii: e004947.

[9] G.D. Lewis, R. Wei, E. Liu, E. Yang, X. Shi, M. Martinovic, et al., Metabolite profiling of blood from individuals undergoing planned myocardial infarction reveals early markers of myocardial injury, J. Clin. Invest. 118 (10) (2008 Oct) 3503-3512, https://doi.org/10.1172/JCI35111.

[10] P. Würtz, A.S. Havulinna, P. Soininen, T. Tynkkynen, D. Prieto-Merino, et al., Metabolite profiling and cardiovascular event risk: a prospective study of 3 population-based cohorts, Circulation 131 (9) (2015 Mar 3) 774-785, https:// doi.org/10.1161/CIRCULATIONAHA.114.013116. Epub 2015 Jan 8.

[11] A. Ganna, S. Salihovic, J. Sundström, C.D. Broeckling, A.K. Hedman, et al., Largescale metabolomic profiling identifies novel biomarkers for incident coronary heart disease, PLoS Genet. 10 (12) (2014 Dec 11) e1004801, https://doi.org/ 10.1371/journal.pgen.1004801 eCollection 2014 Dec

[12] S. Rizza, M. Copetti, C. Rossi, M.A. Cianfarani, M. Zucchelli, et al., Metabolomics signature improves the prediction of cardiovascular events in elderly subjects, 
Atherosclerosis 232 (2) (2014 Feb) 260-264, https://doi.org/10.1016/ j.atherosclerosis.2013.10.029. Epub 2013 Nov 18.

[13] R. Stöhr, B.A. Kappel, D. Carnevale, M. Cavalera, M. Mavilio, et al., TIMP3 interplays with apelin to regulate cardiovascular metabolism in hypercholesterolemic mice, Mol Metab 4 (10) (2015 Aug 6) 741-752, https://doi.org/ 10.1016/j.molmet.2015.07.007 eCollection 2015 Oct.

[14] B.A. Kappel, R. Stöhr, L. De Angelis, M. Mavilio, R. Menghini, M. Federici, Posttranslational modulation of FoxO1 contributes to cardiac remodeling in post-ischemic heart failure, Atherosclerosis 249 (2016 Jun) 148-156, https:// doi.org/10.1016/j.atherosclerosis.2016.04.001.

[15] M. Cardellini, A. Farcomeni, M. Ballanti, M. Morelli, F. Davato, et al., C-peptide: a predictor of cardiovascular mortality in subjects with established atherosclerotic disease, Diabetes Vasc. Dis. Res. 14 (5) (2017 Sep) 395-399, https:// doi.org/10.1177/1479164117710446. Epub 2017 May 31.

[16] A. Bellia, M. Sallì, M. Lombardo, M. D'Adamo, V. Guglielmi, et al., Effects of whole body vibration plus diet on insulin-resistance in middle-aged obese subjects, Int. J. Sports Med. 35 (6) (2014 Jun) 511-516, https://doi.org/ 10.1055/s-0033-1354358. Epub 2013 Nov 13.

[17] M. Mavilio, V. Marchetti, M. Fabrizi, R. Stöhr, A. Marino, et al., A role for Timp3 in microbiota-driven hepatic steatosis and metabolic dysfunction, Cell Rep. 16 (8) (2016 Aug 23) 2269, https://doi.org/10.1016/j.celrep.2016.07.078. Epub 2016 Aug 29.

[18] R. Stöhr, B.A. Kappel, D. Carnevale, M. Cavalera, M. Mavilio, et al., TIMP3 interplays with apelin to regulate cardiovascular metabolism in hypercholesterolemic mice, Mol Metab 4 (10) (2015 Aug 6) 741-752, https://doi.org/ 10.1016/j.molmet.2015.07.007 eCollection 2015 Oct.

[19] J. Xia, D.S. Wishart, Using MetaboAnalyst 3.0 for Comprehensive Metabolomics Data Analysis Current Protocols in Bioinformatics vol. 55, 2016, p. 14, 10.1-14.10.91.

[20] J. Trygg, S. Wold, Orthogonal projections to latent structures (O-PLS), J. Chemometr. 16 (2002) 119-128, https://doi.org/10.1002/cem.695.

[21] G. D'Antona, M. Ragni, A. Cardile, L. Tedesco, M. Dossena, et al., Branchedchain amino acid supplementation promotes survival and supports cardiac and skeletal muscle mitochondrial biogenesis in middle-aged mice, Cell Metabol. 12 (4) (2010 Oct 6) 362-372, https://doi.org/10.1016/ j.cmet.2010.08.016.

[22] T. Li, Z. Zhang, S.C. Kolwicz Jr., L. Abell, N.D. Roe, et al., Defective branchedchain amino acid catabolism disrupts glucose metabolism and sensitizes the heart to ischemia-reperfusion injury, Cell Metabol. 25 (2) (2017 Feb 7) 374-385, https://doi.org/10.1016/j.cmet.2016.11.005.

[23] B.A. Kappel, M. Lehrke, K. Schütt, A. Artati, J. Adamski, et al., Effect of empagliflozin on the metabolic signature of patients with type 2 diabetes mellitus and cardiovascular disease, Circulation 136 (2017) 969-972. Available from: http://circ.ahajournals.org/lookup/doi/10.1161/CIRCULATIONAHA. 117.029166.

[24] L.I. Woolf, C. Hasinoff, A. Perry, Estimation of branched-chain alpha-keto acids in blood by gas chromatography, J. Chromatogr., A 231 (2) (1982 Sep 10) 237-245.

[25] J.B. Brooks, J.V. Kasin, D.M. Fast, M.I. Daneshvar, Detection of metabolites by frequency-pulsed electron capture gas-liquid chromatography in serum and cerebrospinal fluid of a patient with Nocardiainfection, J. Clin. Microbiol. 25 (2) (1987 Feb) 445-448.

[26] T. Tamura, Y. Oki, A. Yoshida, T. Kuriyama, H. Kawakami, et al., Noncompetitive, reversible inhibition of aminoacylase- 1 by a series of L-alpha-hydroxyl and L-alpha-fluoro fatty acids: ligand specificity of aspergillus oryzae and porcine kidney enzymes, Arch. Biochem. Biophys. 379 (2) (2000 Jul 15) $261-266$.

[27] D. Ngo, S. Sinha, D. Shen, E.W. Kuhn, M.J. Keyes, et al. Aptamer-based proteomic profiling reveals novel candidate biomarkers and pathways in cardiovascular disease, Circulation 134 (4) (2016 Jul 26) 270-285.

[28] G. Daniele, R. Guardado Mendoza, D. Winnier, T.V. Fiorentino, Z. Pengou, J. Cornell, F. Andreozzi, C. Jenkinson, E. Cersosimo, M. Federici, D. Tripathy, F. Folli, The inflammatory status score including IL-6, TNF- $\alpha$, osteopontin, fractalkine, MCP-1 and adiponectin underlies whole-body insulin resistance and hyperglycemia in type 2 diabetes mellitus, Acta Diabetol. 51 (1) (2014 Feb) $123-131$.

[29] S. Del Turco, M. Gaggini, G. Daniele, G. Basta, F. Folli, R. Sicari, A. Gastaldelli, Insulin resistance and endothelial dysfunction: a mutual relationship in cardiometabolic risk, Curr. Pharmaceut. Des. 19 (13) (2013) 2420-2431.

[30] P. Welsh, N. Rankin, Q. Li, P.B. Mark, P. Würtz, M. Ala-Korpela, M. Marre, N. Poulter, P. Hamet, J. Chalmers, M. Woodward, N. Sattar, Circulating amino acids and the risk of macrovascular, microvascular and mortality outcomes in individuals with type 2 diabetes: results from the advance trial, Diabetologia (2018 May 4), https://doi.org/10.1007/s00125-018-4619-x.

[31] P. Würtz, A.S. Havulinna, P. Soininen, T. Tynkkynen, D. Prieto-Merino, T. Tillin, A. Ghorbani, A. Artati, Q. Wang, M. Tiainen, A.J. Kangas, J. Kettunen, J. Kaikkonen, V. Mikkilä, A. Jula, M. Kähönen, T. Lehtimäki, D.A. Lawlor, T.R. Gaunt, A.D. Hughes, N. Sattar, T. Illig J. Adamski, T.J. Wang, M. Perola, S. Ripatti, R.S. Vasan, O.T. Raitakari, R.E. Gerszten, J.P. Casas, N. Chaturvedi, M. Ala-Korpela, V. Salomaa, Metabolite profiling and cardiovascular event risk: a prospective study of 3 population-based cohorts, Circulation 131 (9) (2015 Mar 3) 774-785. 